\title{
Major Risk Factors in Head and Neck Cancer: A Retrospective Analysis of 12-Year Experiences
}

\author{
Anil Kumar Dhull, b, Rajeev Atria, Rakesh Dhankhara, \\ Ashok K. Chauhan ${ }^{\text {a }}$, Vivek Kaushal ${ }^{\mathrm{a}}$
}

\begin{abstract}
Background: Head and neck cancer (HNC) is the seventh most common type of cancer in the world and constitute $5 \%$ of the entire cancers worldwide. The global burden of HNC accounts for 650,000 new cases and 350,000 deaths worldwide every year and a major proportion of regional malignancies in India. More than $70 \%$ of squamous cell carcinoma of the head and neck are estimated to be avoidable by lifestyle changes, particularly by effective reduction of exposure to well-known risk factors such as tobacco smoking and alcohol drinking.
\end{abstract}

Methods: A retrospective analysis of 12 years (2001 - 2012) of HNC patients attending RCC, PGIMS Rohtak was done. Total numbers of cancer patients seen were 26,295 and out of these 9,950 patients were of HNCs, which were retrospectively analyzed for their associated risk factors in different $\mathrm{HNC}$ subtypes. Most of the patients, i.e. $92.3 \%$, were presented as locally advanced HNC (stages III and IV).

Results: It has been observed that smoking and alcohol are the strongest independent risk factors responsible for increased risk of HNC and are further having synergetic correlations.

Conclusion: The present study confirms the principal role of alcohol consumption and smoking in HNC carcinogenesis, as well as the differential associations with HNC subtypes, and a significant, positive, multiplicative interaction with different risk factors.

Keywords: Head and neck cancer (HNC); Alcohol; Smoking; Carcinogen; Risk factors; LAHNC; Lifestyle

\section{Introduction}

Worldwide, head and neck cancer (HNC) is the seventh most

Manuscript submitted April 4, 2018, accepted May 10, 2018

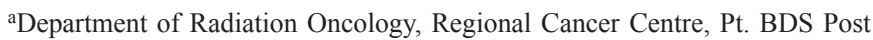
Graduate Institute of Medical Science, Rohtak, Haryana, India

${ }^{\mathrm{b} C}$ Corresponding Author: Anil Kumar Dhull, PO Box: 100, GPO Rohtak-124001, India.Email: anilkdhull@gmail.com

doi: https://doi.org/10.14740/wjon1104w common type of cancer and more than $70 \%$ of squamous cell carcinoma of these types are estimated to be avoidable by lifestyle changes, particularly by effective reduction of exposure to well-known risk factors such as tobacco smoking and alcohol drinking [1-3]. In this large retrospective analysis of 9,950 $\mathrm{HNC}$ patients, different risk factors of $\mathrm{HNC}$ and its subtypes were analyzed and it was observed that smoking is the strongest independent risk factor responsible for increased risk of $\mathrm{HNC}$ and further, this is having synergetic correlations with alcohol consumption.

\section{Materials and Methods}

A retrospective analysis of 12 years from year 2001 - 2012, of HNC patients attending Regional Cancer Centre, PGIMS Rohtak was done. Total numbers of cancer patients seen were 26,295 and out of these 9,950 patients were of HNCs, which were retrospectively analyzed for their associated risk factors in different $\mathrm{HNC}$ subtypes.

In a $\mathrm{HNC}$ population of 9,950 patients, we have analyzed the association of $\mathrm{HNC}$ with smoking in $89 \%$ smokers and $59 \%$ alcoholics of our hospital-based retrospective study. Statistical analysis was carried out using Stata 9.0 and variables were compared using Chi-square test/Fisher's exact test and quantitative characteristics were compared using Student's $t$ test for independent samples/Wilcoxon rank-sum test.

\section{Results}

This is a hospital-based retrospective study of 12 years participating all patients in Regional Cancer Centre, PGIMS Rohtak from the year 2001 to 2012 . Total 26,295 cancer patients were analyzed, out of which, 9,950 (37.8\%) patients were of $\mathrm{HNC}$, with a predominance of male population as $8,686(87 \%)$, and females were $1,264(13 \%)$.

It has been observed that smoking and alcohol are the strongest independent risk factors responsible for increased risk of $\mathrm{HNC}$ and are further having synergetic correlations. Among all the patients of HNCs, men were predominant with male to female ratio of 7:1. It was observed that most common presenting complaints were dysphagia 3,655 (36.7\%), odynophagia $1,876(18.96 \%)$ and cervical swelling 1,843 $(18.5 \%)$ (Table 1). It was also observed that most common age of presentation was the fifth decade of life $(3,091$ pa- 
Table 1. Clinical Presentation of HNC Patients $(n=9,950)$

\begin{tabular}{llll}
\hline Sr. no. & Clinical presentation & Total number of patients $(\mathbf{n}=\mathbf{9 , 9 5 0 )}$ & Percentage of patients $(\mathbf{\%})$ \\
\hline 1 & Bleeding from mouth & 63 & 0.63 \\
\hline 2 & Hoarseness of voice & 944 & 9.48 \\
\hline 3 & Dysphagia & 3,655 & 36.7 \\
\hline 4 & Odynophagia & 1,876 & 18.9 \\
\hline 5 & Ulcerative growth in mouth & 585 & 5.87 \\
\hline 6 & Throat pain & 343 & 3.44 \\
7 & Neck/cervical swelling & 1,843 & 18.5 \\
\hline 8 & Dyspnea & 160 & 1.60 \\
\hline 10 & Trismus & 185 & 1.85 \\
\hline
\end{tabular}

tients) and next common age group was 41-50 years (2,783 patients) and median age of presentation was 54 years (Table 2). Most of the patients, i.e. $92.3 \%$, presented as locally advanced HNC (LAHNC stages III and IV) (Table 3). In this analysis, we have attributed the association of $\mathrm{HNC}$ with smoking and alcohol in 89\% smokers and 59\% alcoholics respectively (Table 4 ).

In this hospital-based retrospective analysis of 9,950 HNC patients spanning over a duration of 12 years, data revealed that out of the total population of HNC ( $\mathrm{n}=9,950) ; 8,250(93 \%)$ were male; while 609 (7\%) were female. Further analysis have shown that in this defined population of $9,950 \mathrm{HNC}$ patients, $89 \%(8,859)$ patients were total smokers, i.e. (smokers only + smokers as well as alcoholics) out of which 3,605 (36\%) patients were smokers only. In this analysis, 5,861 (59\%) were total alcoholics i.e. (alcoholic only + smokers as well as alcoholics) out of which 607 (6\%) were alcoholics only. Patients who were consuming both types, i.e. alcohol and smoking, were 5,254 (53\%) (Fig. 1).

The presented data have shown a strong association of HNC with smoking and hypothesized the effect of socioeconomic position on HNC in the third-world countries, which is in part explained by differential patterns of smoking across social strata. Among alcohol consumers and smoking, men mostly were found predominant consumers for the alcohol and smoking. It was observed that the strength of the association,

Table 2. Age-Wise Distribution of HNC Patients $(n=9,950)$

\begin{tabular}{|lll|}
\hline Age groups (years) & $\begin{array}{l}\text { Number of } \\
\text { HNC patients }\end{array}$ & $\begin{array}{l}\text { Percentage of } \\
\text { patients (\%) }\end{array}$ \\
\hline$<20$ & 40 & 0.4 \\
$21-30$ & 241 & 2.4 \\
\hline $31-40$ & 1,127 & 11.3 \\
$41-50$ & 2,783 & 27.9 \\
$51-60$ & 3,091 & 31 \\
$61-70$ & 2,055 & 20.6 \\
$>70$ & 613 & 6.2 \\
\hline
\end{tabular}

however, differed between different HNC subtypes, i.e. oropharyngeal HNC group (35\%) was most strongly associated with alcohol consumption, while oral cavity, hypopharynx and larynx $\mathrm{HNC}$ group contributed to $7.6 \%, 5 \%$ and $10 \%$, respectively. For HNC overall, an associative synergetic interaction between alcohol consumption and smoking was found.

It was also observed that only 910 out of $5,861(15.5 \%)$ total alcoholic HNC patients (alcoholics only + smokers as well as alcoholics) were having without evidence of disease (NED) status at last follow-up, while 815 out of $4089(20 \%)$ non-alcoholic HNC patients were having NED status at last follow-up. In this analysis on different risk factors of $\mathrm{HNC}$ and its subtypes, we have attributed the association of HNC with smoking and alcohol in 89\% smokers and 59\% alcoholics respectively and observed that smoking is the strongest independent associated risk factor, and alcohol consumption and others are the associated risk factors responsible for increased risk of HNC overall.

\section{Discussion}

HNC includes several malignancies that originate in the paranasal sinuses, nasal cavity, salivary glands, oral cavity, nasopharynx, oropharynx, hypopharynx and larynx [4, 5]. "Head and neck cancer" usually refers to neoplasms arising from below the skull base to the region of thoracic inlet. HNC is the seventh most common type of cancer in the world and constitute $5 \%$ of the entire cancers worldwide [1-5]. The global burden of $\mathrm{HNC}$ accounts for 650,000 new cases and 350,000

Table 3. Stage-Wise Distribution of HNC Patients $(n=9,950)$

\begin{tabular}{lll}
\hline $\begin{array}{l}\text { Stage-wise } \\
\text { distribution }\end{array}$ & $\begin{array}{l}\text { Number of } \\
\text { HNC patients }\end{array}$ & $\begin{array}{l}\text { Percentage of } \\
\text { patients }(\%)\end{array}$ \\
\hline Stage I & 180 & 1.8 \\
Stage II & 591 & 5.9 \\
Stage III & 2,844 & 28.6 \\
Stage IV & 6,335 & 63.7 \\
\hline
\end{tabular}


Table 4. Smoker and Alcoholic Population With Different HNC Subtypes $(n=9,950)$

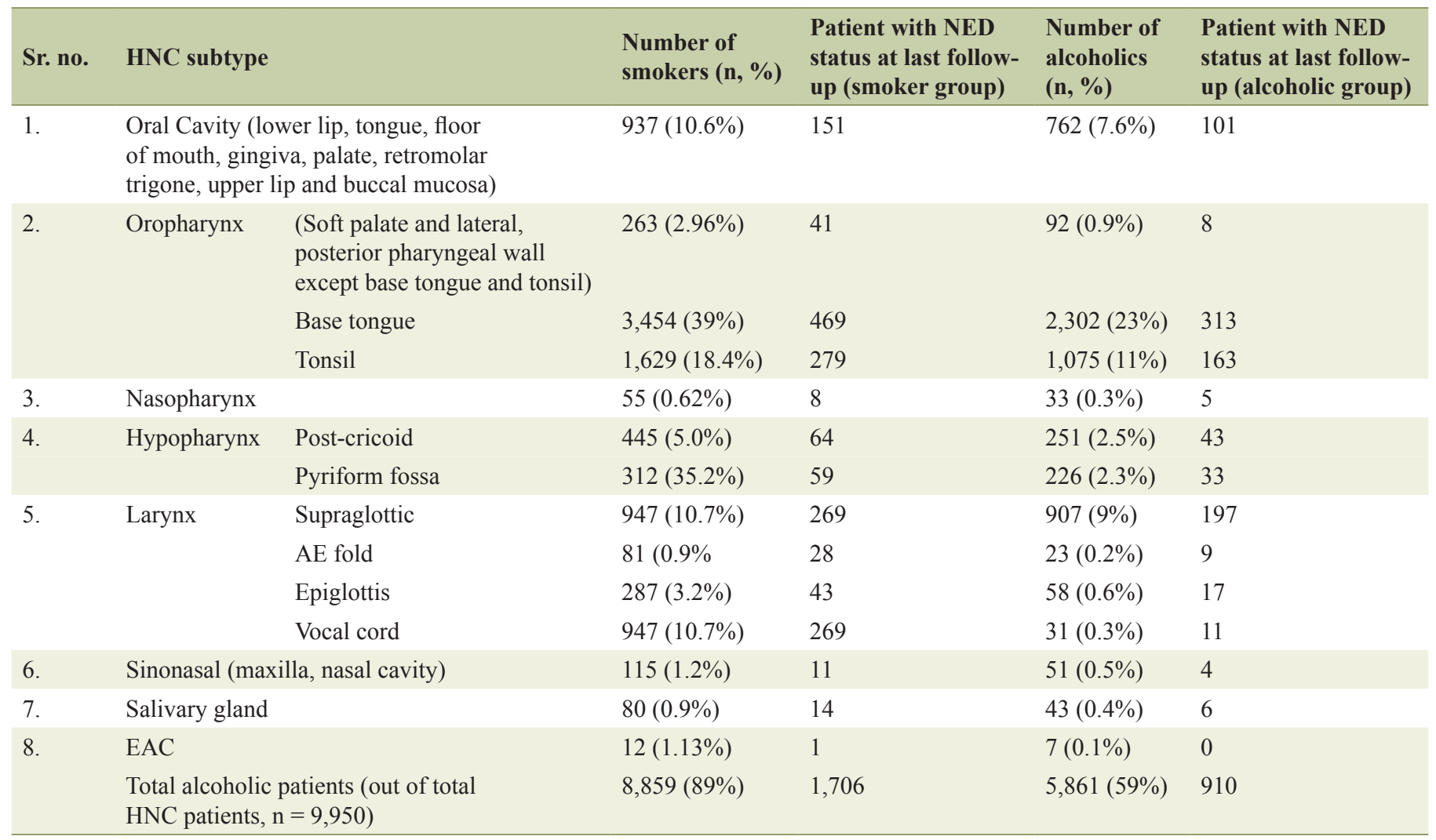

deaths worldwide every year and a major proportion of regional malignancies in India [1-3]. According to hospital-based cancer registries in India, HNCs account for $29.8-50.4 \%$ of all

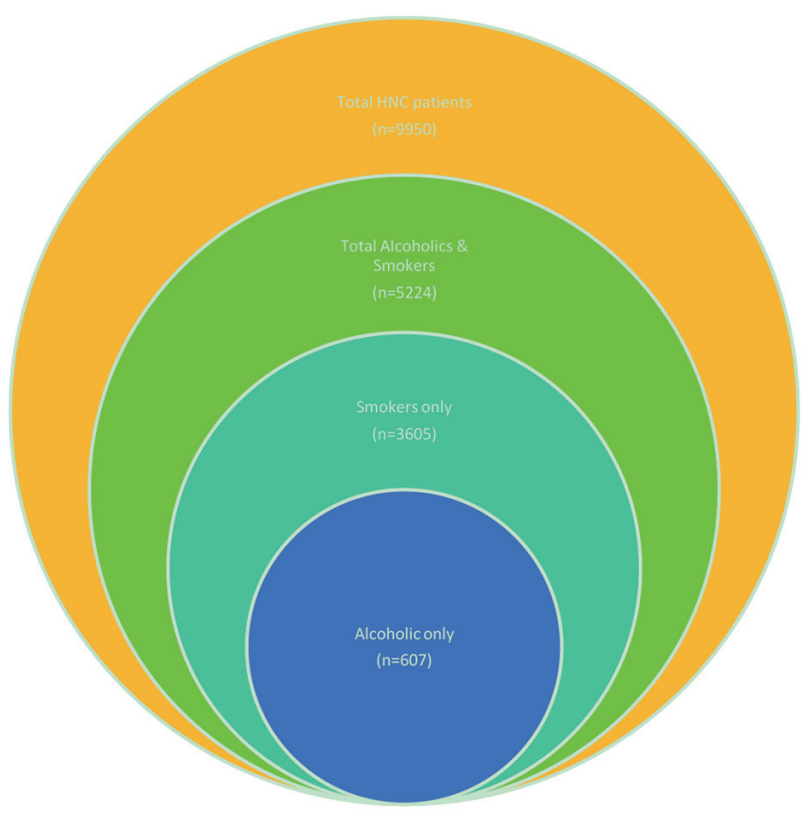

Figure 1. Relationship between "smokers", "alcoholics", "alcoholics and smokers" and "total HNC patients" represented through Venn diagram. cancers in males and $11-22 \%$ of all cancers in females [1-4]. The incidence of HNC in our institute in the past 20 years constitutes one-third of all the malignancies [6]. Epithelial carcinomas of head and neck arise from the mucosal surfaces and typically are squamous cell in nature and these squamous cell carcinomas (SCCs) account for more than $90 \%$ of HNCs. Locally advanced cancers of the mouth and pharynx, or throat, refer to cancer that has spread locally outside the site of cancer origin $[2,4]$.

Although the exact pathogenesis of HNC is not yet fully elucidated, there are ample evidences suggesting that HNCs are complex multifactorial disorders involving genetic factors, lifestyle and environmental factors $[7,8]$ and some low-penetrant genes have been identified as potential HNC susceptibility genes [2, 9-10].

Cigarette smoking is a well-established risk factor for $\mathrm{HNC}$ with a well-defined dose-response relationship for duration and frequency of use [1-3]. This retrospective analysis confirms the strong associations of tobacco (bidi/cigarette) smoking with increased risk of $\mathrm{HNC}$ overall and its subtypes. Among different subtypes, however, oropharyngeal carcinoma was least associated with smoking in any form; besides these, smoking status, frequency and duration of smoking, all appear to be important associates between smoking and risk of HNC overall [3]. As seen in some meta-analysis, these results are consistent with the existing literature showing that smoking has a stronger effect on the larynx and pharynx than on the oral cavity $[8,9,11,12]$. The reason for this could be 
the aerodynamics of respiratory flow in the upper airway: this flow changes from laminar in the oral cavity to turbulent in the larynx, which may result in the larynx and pharynx having a higher exposure to inhaled air and thus to smoke than the oral cavity.

As evident from this analysis and other meta-analysis, around $90 \%$ of all HNCs occur in people who smoke tobacco alone or with alcohol $[2,8-10,12,13]$. The longer a person smokes and the more tobacco they consume the greater their risk of developing these cancers $[2,11]$. For male and female smokers, the average risk for developing $\mathrm{HNC}$ is ten and five times higher, respectively, compared to lifetime non-smokers $[2,11]$. Heavy smokers also have up to 20 times the risk of developing laryngeal cancer compared to lifetime non-smokers. Quitting smoking can significantly reduce this risk. After about 5 years of quitting the risk of developing oral and pharyngeal cancers is halved $[2,9,11]$.

Alcohol consumption and tobacco (whether smoked, chewed or inhaled as snuff) and dietary factors, are established risk factors for HNC originating from the oral cavity, pharynx and larynx, and are likely to be differentially associated with risk of those HNC subtypes [9, 10, 11, 13]. Our results are in accordance with the existing literature, showing alcohol consumption to be an independent as well as associated risk factor for the development of $\mathrm{HNC}$, with a strong, dose-response relationship [2, 8-10, 12, 13]. Alcoholic beverages and acetaldehyde, the main metabolite of ethanol, are classified as a class I carcinogen [14]. There is a probability that alcohol after being metabolized acts both directly and indirectly in $\mathrm{HNC}$ carcinogenesis, the latter for example by acting as a solvent for other possible carcinogens, such as tobacco carcinogens $[11,13]$.

There are theories which hypothesized that 1) alcohol consumption and cigarette smoking are strongly, positively associated with HNC risk, with multiplicative interaction; 2) alcohol and tobacco consumption are synergistic to each other; 3 ) these risks are different for oral cavity cancer (OCC), oro-/ hypopharyngeal cancer and laryngeal cancer [13].

Our present retrospective study confirms a multiplicative interaction between alcohol consumption and smoking in HNC overall [2, 8-10, 12, 13]. The interaction effect between alcohol consumption and smoking is biologically conceivable, since alcohol can act as a solvent for carcinogens in cigarette smoke and make the mucosa more permeable for these carcinogens; as a result, the carcinogenic properties of both factors are likely to be enhanced in the presence of one another $[8,9,11]$. The principal risk factors for this disease include tobacco and alcohol use, exposure to the human papillomavirus (HPV) contributes to the development of at least $90 \%$ of squamous cell carcinoma of the head and neck (SCCHN) cases in a growing younger population $[8,15]$. It has been reported that HNC is much more common in smokers than in non-smokers and most common in males over 50 years of age [12]. In recent years, evidence has accumulated to support the hypothesis that diet may also play an important etiological role in development of the disease, and $10-15 \%$ of SCCHN cases in Europe are associated with a low intake of fruit and vegetables [16-18].

For HNC overall, increased risks were found for every exposure combination of alcohol consumption and cigarette smoking, mostly statistically significantly, compared to never smokers and abstainers. In literature, a statistically significant, positive, multiplicative interaction was found between alcohol consumption group and cigarette smokers $[2,8,13]$. The other factors are poor oral hygiene, chronic mechanical irritation in case of oral cavity cancers. Squamous cell carcinoma may also develop from premalignant conditions such as submucous fibrosis, which in return may be a result of tobacco or betel nut chewing.

There is increasing evidence that viruses may contribute to causation of HNCs. Infection with the Epstein-Barr virus is clearly associated with nasopharyngeal carcinoma and DNA from the human papilloma virus has also been detected in $\mathrm{HNC}[15,16]$. The clinical manifestations of disease vary according to the stage and primary site of tumor. However, dysphagia, odynophagia, otalgia, hoarseness, mucosal ulceration, decreased tongue mobility, nasal obstruction, epistaxis, cranial nerve neuropathies and cervical lymphadenopathy are common presenting complaints. The advanced stages of all HNCs have easily detectable signs and symptoms, including trismus, formation of fistulae, etc.

In preliminary studies, patients who were HPV-positive and were smokers at diagnosis were reported to have worse survival than those who were not, possibly because of an increased risk for both local recurrence and distant metastases $[2,8-10,12,13]$. In a recent analysis, by found that tumor HPV status and tobacco smoking were the two strongest, independent determinants of progression free survival (PFS) and overall free survival (OS) for patients with oropharyngeal cancer (OPC) treated by chemoradiation [18]. Browman et al originally reported that smoking during radiotherapy reduced response rates and 2-year survival for HNC patients and also smoking during radiation therapy has been reported to increase the severity of mucositis, thus increasing the frequency of treatment breaks for smokers, which are known to decrease disease control [19] (although not observed in this study).

Management of head and neck carcinoma has come a long way from the era of primitive surgery to advanced and state of the art treatment by image-guided radiation therapy (IGRT). Similarly, the diagnostic workup has evolved from the crude staging by only clinical methods to sophisticated and logically convincing biological imaging. History with emphasis on alcohol intake, smoking, tobacco chewing, general physical examination, examination of oral cavity, oropharynx, nasopharynx, laryngopharynx (direct and indirect laryngoscopy), and examination of the lymph nodes and biopsy of any suspected areas is preliminary to confirm and stage HNC.

\section{Conclusions}

The present study, confirms the principal role of alcohol consumption and smoking in HNC carcinogenesis, as well as the differential associations with HNC subtypes, and a significant, positive, multiplicative interaction with different risk factors. As the existing evidence is largely based on retrospective analysis, this contributes to establish the association between alco- 
hol consumption and smoking with risk of $\mathrm{HNC}$ overall and, more specifically different HNC subtypes.

\section{Acknowledgments}

We are thankful for our Departmental team for their help in collection of data and statistical analysis.

\section{Conflicts of Interest}

There is no conflict of interest.

\section{Abbreviations}

DNA: Deoxyribonucleic acid; EBV: Epstein-Barr virus; HNC: Head and neck cancer; HPV: Human papilloma virus; IGRT: Image-guided radiation therapy; LAHNC: Locally advanced head and neck cancer; OCC: Oral cavity cancer; OPC: Oropharyngeal cancer; OS: Overall survival; PFS: Progression free survival; RCC: Regional cancer centre; SCC: Squamous cell carcinomas; SCCHN: Squamous cell carcinoma of the head and neck

\section{References}

1. Ferlay J, Soerjomataram I, Ervik M, Dikshit R, Eser S, Mathers C. GLOBOCAN 2012 v1.0, Cancer Incidence and Mortality Worldwide: IARC Cancer Base No. 11 [Internet]. Lyon, France: International Agency for Research on Cancer Available from http://globocan.iarc.fr. 2012.

2. Gregory TW, Scott ML, George EL, Waun KH. Head and neck cancer In: James FH, Emil FN, Robert CB, Donald WK, Donald LM, Ralph RW, editors. Cancer Medicine. Philadelphia: Lea and Febiger; 1993;1211-1274.

3. Cogliano VJ, Baan R, Straif K, Grosse Y, Lauby-Secretan B, El Ghissassi F, Bouvard V, et al. Preventable exposures associated with human cancers. J Natl Cancer Inst. 2011;103(24):1827-1839.

4. Vokes EE. Head and neck cancer In: Fauci AS, Braunwald E, Isselbacher KJ, Wilson JD, Maltin JB, Kasper DL, et al, editors. Harrison's Principles of Internal Medicine. New York: Mc Graw Hill. 2005;16:503-506.

5. Rath GK, Chaudhary K, Mohanti BK. Introduction In: Rath GK, Mohanti BK, editors. Textbook of radiation oncology, principles and practice. New Delhi: B.I. Churchilll Livingstone. 2000:1-24.

6. Das BP. Cancer patterns in Haryana: twenty one years' experience. Radiation Oncology. 2005; 5(1):22-32.

7. Abusail MS, Dirweesh AM, Salih RA, Gadelkarim AH. Expression of EGFR and p53 in head and neck tumors among Sudanese patients. Asian Pac J Cancer Prev. 2013;14(11):6415-6418.

8. Maasland DH, van den Brandt PA, Kremer B, Goldbohm RA, Schouten LJ. Alcohol consumption, cigarette smoking and the risk of subtypes of head-neck cancer: results from the Netherlands Cohort Study. BMC Cancer. 2014;14:187.

9. International Agency for Research on Cancer, World Health Organization. Alcohol drinking. Lyon: World Health Organization, International Agency for Research on Cancer. 1988; ISBN 92-832-1244-4:8.

10. Poschl G, Seitz HK. Alcohol and cancer. Alcohol Alcohol. 2004;39(3):155-165.

11. Boing AF, Antunes JL, de Carvalho MB, de Gois Filho JF, Kowalski LP, Michaluart P, Jr., Head, et al. How much do smoking and alcohol consumption explain socioeconomic inequalities in head and neck cancer risk? J Epidemiol Community Health. 2011;65(8):709-714.

12. Kamangar F, Dores GM, Anderson WF. Patterns of cancer incidence, mortality, and prevalence across five continents: defining priorities to reduce cancer disparities in different geographic regions of the world. J Clin Oncol. 2006;24(14):2137-2150.

13. Dhull AK, Atri R, Kaushal V, Malik G, Soni A, Dhankhar $\mathrm{R}$, Chauhan A. Alcohol as a risk factor in HNC, an enormous toll on the lives and communities. J Evid Based Med Healthc. 2016;3(12):354-360.

14. Green FL, Page DL, Fleming ID. editors. AJCC Cancer Staging Manual 6th ed. New York: Springer-Verlag. 2002;6:32-34.

15. Fathraeus R, Fu HL, Ernbrg I. Expression of Epstein Barr virus encoded protein in nasopharyngeal carcinoma. Int J Cancer. 1998;42:329.

16. Babiker AY, Eltom FM, Abdalaziz MS, Rahmani A, Abusail S, Ahmed HG. Screening for high risk human papilloma virus (HR-HPV) subtypes, among Sudanese patients with oral lesions. Int J Clin Exp Med. 2013;6(4):275-281.

17. Dhull AK, Bansal N, Atri R, Chauhan AK, Kaushal V. The integrated relations of fruits and vegetables with cancer. J Cancer Prev Curr Res. 2015;2(5):00053.

18. D'Souza G, Kreimer AR, Viscidi R, Pawlita M, Fakhry C, Koch WM, Westra WH, et al. Case-control study of human papillomavirus and oropharyngeal cancer. N Engl J Med. 2007;356(19):1944-1956.

19. Browman GP, Wong G, Hodson I, Sathya J, Russell R, McAlpine L, Skingley $P$, et al. Influence of cigarette smoking on the efficacy of radiation therapy in head and neck cancer. N Engl J Med. 1993;328(3):159-163. 